\title{
Crystal structures of Thiamine monophosphate kinase from Acinetobacter baumannii in complex with substrates and products
}

Amy H. Sullivan, David M. Dranow, Peter S. Horanyi, Don D. Lorimer, Thomas E. Edwards*, Jan Abendroth*

Seattle Structural Genomics Center for Infectious Disease, Beryllium Discovery

Acinetobacter baumanii is a member of the ESKAPE pathogens (Enterococcus faecium, Staphylococcus aureus, Klebsiella pneumonia, Acinetobacter baumanii, Pseudomonas aeruginosa, and Enterobacter species), all of which have a high rate of antibiotic resistance, are opportunistic, and are responsible for a large number of hospital borne infections. Therefore, A. baumanii is one of the target organisms for SSGCID. Thiamine monophosphate kinase (ThiL) catalyzes the last step of the thiamine pyrophosphate synthesis, the ATP-dependent phosphorylation of Thiamine monophosphate (TMP) to Thiamine pyrophosphate (TPP). Humans lack ThiL, which might make ThiL an attractive drug target.

We solved the structure of $A$. baumanii ThiL in complex with its substrates TMP / AMPPNP, and in complex with its products TPP / ADP. High resolution of the data and anomalous diffraction allows for a detailed description of the binding mode of substrates and products, and the metal environment. The structures highlight the path of the reaction, and a distinct variability of metal content. 